\title{
FRAÇÕES DE FÓSFORO NO SOLO E SUA RELAÇÃO COM A ABSORÇÃO PELAS PLANTAS DE ARROZ IRRIGADO POR ALAGAMENTO EM SOLOS DO RIO GRANDE DO SUL ${ }^{(1)}$
}

\author{
Gustavo Krüger Gonçalves ${ }^{(2)} \&$ Egon José Meurer ${ }^{(3)}$
}

\begin{abstract}
RESUMO
Os solos utilizados na cultura de arroz irrigado, por alagamento, no Estado do Rio Grande do Sul são oriundos de diferentes materiais de origem, os quais conferem variações na distribuição das frações de $P$. Com o objetivo de quantificar as frações de $P$ no solo e de relacioná-las com o $P$ acumulado por plantas de arroz, foi realizado um experimento em casa de vegetação com amostras de quatro solos, nos quais foi cultivado arroz por 35 dias, sem e com a adição de $39,30 \mathrm{mg} \mathrm{kg}^{-1}$ de $P$. Nas amostras dos solos antes do cultivo, foi extraído o $\mathrm{P}$ disponível com o extrator Mehlich-1 e feito o fracionamento de formas de $P$ nas amostras pelo método de Hedley. Os teores de $P$ acumulados pelas plantas de arroz correlacionaram-se mais estreitamente com as frações de P que estimaram o P lábil (Pi RTA e Pi NaHCO ${ }_{3}$ ) bem como com as frações que estimaram o $\mathrm{P}$ moderadamente lábil $\left(\mathrm{NaOH} 0,1 \mathrm{~mol} \mathrm{~L}^{-1}\right.$ e $\mathrm{NaOH} \mathrm{0,5} \mathrm{mol} \mathrm{L}^{-1}$ ). Os teores de $\mathrm{P}$ extraídos das amostras dos solos pela resina e por Mehlich-1 correlacionaram-se com os teores de $\mathbf{P}$ extraídos das frações lábil e moderadamente lábil, sendo o grau de associação com essas frações maior para a resina (Pi RTA). O P que foi recentemente adicionado às amostras dos solos na forma de superfosfato triplo incrementou principalmente a fração moderadamente lábil ( $\mathrm{Pi} \mathrm{NaOH} 0,1 \mathrm{~mol} \mathrm{~L}^{-1}$ ) e, em menor proporção, as frações que compõem o $\mathrm{P}$ lábil do solo (Pi $\mathrm{NaHCO}_{3}$ e Pi RTA).
\end{abstract}

Termos de indexação: fracionamento de fósforo, disponibilidade de fósforo, solos alagados.

\footnotetext{
(1) Parte da Tese de Doutorado do primeiro autor apresentada ao Programa de Pós-Graduação em Ciência do Solo da Universidade Federal do Rio Grande do Sul - UFRGS. Recebido para publicação em dezembro de 2007 e aprovado em fevereiro de 2009.

(2) Professor da Universidade Federal do Rio Grande do Sul - UFRGS. Av. Bento Gonçalves 7712, CEP 91540-000 Porto Alegre (RS). E-mail: gutosolos@ibest.com.br

${ }^{(3)}$ Professor do Departamento de Solo e do Programa de Pós-graduação em Ciência do Solo, UFRGS. Bolsista do CNPq. E-mail: egon.meurer@ufrgs.br
} 


\title{
SUMMARY: PHOSPHORUS FRACTIONS AND THEIR RELATIONSHIP WITH PHOSPHORUS UPTAKE BY FLOODED RICE IN RIO GRANDE DO SUL
}

\begin{abstract}
Soils used for flooded rice in the State of Rio Grande do Sul, Brazil, evolved from different materials which led to variations in the distribution of $P$ fractions. In order to quantify the phosphorus fractions and relate them with the Puptake by rice plants, a greenhouse experiment was conducted with four soil samples where rice was grown for 35 days, without and with the addition of $39.30 \mathrm{mg} \mathrm{kg}^{-1} \mathrm{P}$. In the soil samples before cultivation, the available P was determined (Mehlich-1) and the fractionation of P in the samples was determined by the Hedley's method. The P uptake by rice plants was correlated with the labile P fractions ( $\mathrm{Pi}$ RTA and $\mathrm{Pi} \mathrm{NaHCO}$ ) and correlated also with the moderately labile fractions ( $\mathrm{NaOH} 0.1 \mathrm{~mol} \mathrm{~L}^{-1}$ and 0.5 $\left.\mathrm{NaOH} \mathrm{mol} L^{-1}\right)$. The Pextracted from the soil samples by resin and Mehlich-1 correlated with the Pextracted by labile and moderately labile fractions; the association was stronger for resin (Pi RTA). The P added most recently to the soil samples as triple superphosphate increased mainly the fraction of moderately labile $\mathrm{P}\left(\mathrm{Pi} \mathrm{NaOH} 0.1 \mathrm{~mol} \mathrm{~L}^{-1}\right)$ and, to a lesser extent, the labile fraction ( $\mathrm{Pi} \mathrm{NaHCO}_{3}$ and $\mathrm{Pi} \mathrm{RTA}$ ).
\end{abstract}

Index terms: phosphorus fractionation, phosphorus availability, wetland soils.

\section{INTRODUÇÃO}

As recomendações para a adubação fosfatada para a cultura do arroz irrigado, por alagamento, no Estado do Rio Grande do Sul foram baseadas em experimentos de calibração em Planossolos, utilizando-se o extrator Mehlich-1 para avaliar o P disponível. A eficiência desse método em estimar o $\mathrm{P}$ disponível para essa cultura pode ser questionada, já que as frações desse elemento extraídas por esse método podem não ser as mesmas absorvidas pelas plantas. Grande et al. (1986), em solos de várzea do Estado de Minas Gerais, encontraram alta correlação para a resina de troca aniônica e baixa para o extrator Mehlich-1 quando avaliaram a disponibilidade do P para o arroz irrigado. No entanto, Silva (1996) não observou diferenças para esses extratores em solos alagados do Rio Grande do Sul.

Procurar relações entre as diferentes frações de $\mathrm{P}$ no solo com os teores de $\mathrm{P}$ acumulados pelas plantas de arroz pode ser um indicativo da fração ou frações que contribuem para o suprimento de $\mathrm{P}$ para a cultura, o que pode auxiliar na seleção de extratores mais adequados para avaliar a disponibilidade de $\mathrm{P}$ para o arroz irrigado, por alagamento. As técnicas mais usadas para o fracionamento de $\mathrm{P}$ em solos são as de Chang \& Jackson (1957) e de Hedley et al. (1982) modificado por Condron et al. (1985). Este último método utiliza, sequencialmente, extratores de menor a maior poder de dessorção e permite a separação das formas de $\mathrm{P}$ segundo sua natureza (orgânica ou inorgânica) e facilidade de dessorção (Cross \& Schlesinger, 1995). As formas de P extraídas por resina trocadora de ânions (RTA) e por bicarbonato de sódio $\left(\mathrm{NaHCO}_{3}\right)$ são consideradas formas lábeis, de maior disponibilidade para as plantas; as extraídas com $\mathrm{NaOH} 0,1 \mathrm{~mol} \mathrm{~L}^{-1}$, formas moderadamente lábeis; e as frações de $\mathrm{P}$ extraídas por $\mathrm{NaOH} 0,5 \mathrm{~mol} \mathrm{~L}^{-1}$ (Condron et al., 1985), por $\mathrm{HCl} 1 \mathrm{~mol} \mathrm{~L}^{-1} \mathrm{e}$ a fração residual, formas não-lábeis.
Os teores de $\mathrm{P}$ extraído com RTA e o $\mathrm{NaHCO}_{3}$ representam o $\mathrm{P}$ dissolvido da fase sólida em equilíbrio com o P da solução do solo. A fração $\mathrm{Pi} \mathrm{NaOH}$ $0,1 \mathrm{~mol} \mathrm{~L}^{-1}$ representa o P inorgânico moderadamente lábil, quimiossorvido por ligações monodentadas e bidentadas aos óxidos e à caulinita (Hedley et al., 1982), ao passo que a fração orgânica obtida por este extrator representa o $\mathrm{P}$ orgânico moderadamente lábil ligado aos ácidos húmicos As frações $\mathrm{Pi}$ e $\mathrm{Po} \mathrm{NaOH}$ $0,5 \mathrm{~mol} \mathrm{~L}^{-1}$ representam o P inorgânico e orgânico fisicamente protegidos no interior de microagregados. A fração $\mathrm{HCl} 1 \mathrm{~mol} \mathrm{~L}^{-1}$ representa as formas inorgânicas de $\mathrm{P}$ associadas ao Ca. A fração residual representa o $\mathrm{P}$ nas substâncias húmicas, bem como formas inorgânicas de $\mathrm{P}$ insolúveis. $\mathrm{O} \mathrm{P}$ total representa todas as formas, incluindo o $\mathrm{P}$ estrutural (Cross \& Schlesinger, 1995).

No Rio Grande do Sul, os solos utilizados para o cultivo do arroz irrigado, por alagamento, são oriundos de diferentes materiais de origem, o que, provavelmente, resulta em variações na distribuição das formas de P. As hipóteses que nortearam a execução deste trabalho foram: (a) solos oriundos de sedimento de basalto têm maior teor de $\mathrm{P}$ extraído pelos extratores $\mathrm{Pi} \mathrm{NaOH} 0,1 \mathrm{~mol} \mathrm{~L}^{-1}$ e $\mathrm{Pi} \mathrm{NaOH}$ $0,5 \mathrm{~mol} \mathrm{~L}^{-1}$ quando comparados aos solos oriundos de sedimento de granito. Isso se deve aos maiores teores de óxidos de Fe cristalinos e de baixa cristalinidade nos solos oriundos de sedimento de basalto, em relação aos solos oriundos de sedimento de granito; (b) os teores de $\mathrm{P}$ acumulados por plantas de arroz em solos alagados apresentam associação com as frações de $\mathrm{P}$ inorgânicas extraídas pelos extratores Resina, $\mathrm{NaHCO}_{3}, \mathrm{NaOH} 0,1 \mathrm{~mol} \mathrm{~L}^{-1}$ e NaOH 0,5 mol L-1. As formas consideradas moderadamente lábil e não-lábil $\left(\mathrm{NaOH} 0,1\right.$ e 0,5 $\left.\mathrm{mol} \mathrm{L}^{-1}\right)$ são dissolvidas durante a redução do $\mathrm{Fe}$, sendo acessadas pelas plantas de arroz; e (c) os teores de P extraídos pelos extratores Resina e Mehlich-1 apresentam associação com a fração de $\mathrm{P}$ 
inorgânica lábil extraída pelo extrator $\mathrm{NaHCO}_{3}$. Assim, os objetivos deste trabalho foram: quantificar as frações de $\mathrm{P}$ em solos utilizados no cultivo de arroz irrigado por alagamento, utilizando o fracionamento de Hedley, e relacionar essas frações com o P acumulado por plantas de arroz, e relacionar as frações do P obtidas no fracionamento com o P extraído por resina trocadora de íons e com o $\mathrm{P}$ extraído por Mehlich-1.

\section{MATERIAL E MÉTODOS}

O experimento foi realizado em casa de vegetação entre dezembro de 2005 e fevereiro de 2006, com amostras dos seguintes solos coletados no Estado do Rio Grande do Sul: (a) Planossolo Háplico eutrófico solódico, coletado no município de Pelotas; (b) Luvissolo Crômico pálico abrúptico, coletado no município de Itaqui; (c) Vertissolo Ebânico órtico típico, coletado no município de Alegrete; e (d) Chernossolo Ebânico carbonático vértico, coletado no município de Alegrete (Quadro 1). Amostras da camada superficial dos solos $(0-20 \mathrm{~cm})$ foram secas ao ar, destorroadas e passadas em peneira de $2 \mathrm{~mm}$ de malha e colocadas em recipientes de vidro de $2,5 \mathrm{dm}^{3}$ (unidade experimental) na quantidade de $2,2 \mathrm{~kg}$ de solo seco. Nas amostras dos solos foram determinados os teores de óxidos de Fe e de Mn (Mehra \& Jackson, 1960; Schwertmann, 1964), o P disponível (resina trocadora de íons e Mehlich-1) e o fracionamento de $\mathrm{P}$ pelo método de Hedley et al. (1982) modificado por Condron et al. (1985), no qual amostras de $0,5 \mathrm{~g}$ de solo seco foram submetidas à extração sequencial com resina trocadora de ânions (placas AR 103 QDP 434 - fração Pi RTA), $\mathrm{NaHCO}_{3}$ 0,5 mol L-1 (frações $\mathrm{Pi} \mathrm{NaHCO}_{3} \mathrm{e}$ Po $\mathrm{NaHCO}_{3}$ ), NaOH $0,1 \mathrm{~mol} \mathrm{~L}^{-1}$ (frações Pi NaOH $0,1 \mathrm{~mol} \mathrm{~L}^{-1} \mathrm{e}$ Po NaOH 0,1 $\left.\mathrm{mol} \mathrm{L}^{-1}\right), \mathrm{HCl} 1,0 \mathrm{~mol} \mathrm{~L}^{-1}$ (fração Pi HCL) e $\mathrm{NaOH} 0,5 \mathrm{~mol} \mathrm{~L}^{-1}$ (frações Pi

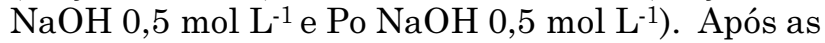
extrações, o solo remanescente foi seco em estufa e submetido à digestão com $\mathrm{H}_{2} \mathrm{SO}_{4}+\mathrm{H}_{2} \mathrm{O}_{2}+\mathrm{MgCl}_{2}$ (fração P Residual), conforme descrito em Brookes \& Powson (1981). O P inorgânico dos extratos alcalinos de $\mathrm{NaHCO}_{3}$ e $\mathrm{NaOH}$ foi determinado pelo método de Dick \& Tabatabai (1977). Esses extratores apresentam altas concentrações de $\mathrm{OH}^{-}$, que proporcionam o deslocamento do $\mathrm{P}$ adsorvido aos óxidos de $\mathrm{Fe}$ e Al. Nesses extratos alcalinos, o P total foi determinado por digestão com persulfato de amônio + ácido sulfúrico em autoclave (USEPA, 1971), sendo o Po (P orgânico) obtido pela diferença entre o $\mathrm{P}$ total e o P inorgânico. O P dos extratos ácidos foi determinado segundo Murphy \& Riley (1962). O mecanismo dos extratores ácidos é baseado na manutenção de uma atividade do íon $\mathrm{H}^{+}$na solução suficiente para dissolução de formas inorgânicas de $\mathrm{P}$ associadas ao Ca. Nas unidades experimentais foram adicionadas duas doses de $\mathrm{P}\left(0\right.$ e $39,30 \mathrm{mg} \mathrm{kg}^{-1}$ de $\left.\mathrm{P}\right)$, utilizando-se como fonte o superfosfato triplo (SFT) previamente moído e passado em peneira de $0,297 \mathrm{~mm}$, para uniformizar o tamanho das partículas. Posteriormente, foram colocadas para germinar oito sementes de arroz cultivar IRGA 417, por recipiente, mantendo-se a umidade gravimétrica em torno de $18 \%$, pela adição de água destilada, até o estádio V3 (Counce et al., 2000). Neste estádio, foi realizado o desbaste, deixando-se três plantas por unidade experimental, e aplicaram-se superficialmente $30 \mathrm{mg} \mathrm{kg}^{-1}$ de $\mathrm{N}$ na forma de ureia. Em seguida, as unidades experimentais foram alagadas, com água destilada, mantendo-se uma lâmina de água de $5,0 \mathrm{~cm}$ de altura, durante 35 dias. Após esse período, a parte aérea das plantas foi coletada a $1,0 \mathrm{~cm}$ da superfície do solo e seca em estufa, à temperatura de $65^{\circ} \mathrm{C}$, por $72 \mathrm{~h}$. Posteriormente à pesagem do material e avaliação da produção de matéria seca, o tecido foi moído e nele determinado o teor de $\mathrm{P}$ conforme Tedesco et al. (1995). O experimento constituiu-se de um fatorial $4 \times 2$, distribuído em blocos ao acaso, com três repetições, onde foram estudados os seguintes fatores: (a) fator solos (Planossolo, Luvissolo, Vertissolo e Chernossolo) e (b) fator doses de $\mathrm{P}\left(0 \mathrm{e} 39,30 \mathrm{mg} \mathrm{kg}^{-1} \mathrm{de} \mathrm{P}\right)$.

\section{Quadro 1. Atributos das amostras dos solos utilizados no experimento}

\begin{tabular}{|c|c|c|c|c|c|c|c|c|c|c|c|}
\hline $\begin{array}{c}\text { Classificação taxonômica } \\
\text { (Embrapa, 2006) }\end{array}$ & $\begin{array}{c}\text { Material } \\
\text { de origem }\end{array}$ & Argila & MO & $\begin{array}{c}\mathrm{pH} \\
\mathrm{H}_{2} \mathrm{O}\end{array}$ & $\mathbf{K}$ & $\mathbf{P}$ & $\begin{array}{l}\text { Saturação } \\
\mathrm{Al}\end{array}$ & $\mathbf{F e}_{\mathbf{o x}}^{(1)}$ & $F \mathbf{e}_{d}^{(2)}$ & $\mathbf{M n}_{\mathrm{ox}}^{(1)}$ & $\mathbf{M n}_{\mathrm{d}}^{(2)}$ \\
\hline & & \multicolumn{2}{|c|}{$-\mathrm{g} \mathrm{kg}^{-1} \_$} & & \multicolumn{2}{|c|}{$-\mathrm{mg} \mathrm{kg}^{\cdot 1}-$} & $\%$ & \multicolumn{4}{|c|}{$\mathrm{g} \mathrm{kg}^{-1}$} \\
\hline $\begin{array}{l}\text { Planossolo Háplico } \\
\text { eutrófico solódico }\end{array}$ & Granito & 200 & 22 & 4,7 & 61 & 3,0 & 8,9 & 0,37 & 5,50 & 0,12 & 1,65 \\
\hline $\begin{array}{l}\text { Luvissolo Crômico } \\
\text { pálico abrúptico }\end{array}$ & Basalto & 230 & 17 & 4,3 & 80 & 2,5 & 13,5 & 0,92 & 8,00 & 0,36 & 2,20 \\
\hline $\begin{array}{l}\text { Vertissolo Ebânico } \\
\text { órtico típico }\end{array}$ & Basalto & 320 & 43 & 5,5 & 102 & 2,9 & 0,0 & 1,35 & 10,70 & 0,49 & 2,20 \\
\hline $\begin{array}{l}\text { Chernossolo Ebânico } \\
\text { carbonático vértico }\end{array}$ & Basalto & 280 & 26 & 4,9 & 114 & 2,7 & 3,1 & 1,45 & 10,80 & 0,70 & 2,60 \\
\hline
\end{tabular}

(1) Extração com oxalato de amônio a pH6; (2) extração com ditionito-citrato-bicarbonato. 
Os teores de $\mathrm{P}$ obtidos em cada extrator (variável $\mathrm{x}$ ) e os teores de $\mathrm{P}$ acumulados pelas plantas de arroz (variável y) foram submetidos à regressão linear. Os teores de $\mathrm{P}$ obtidos pelos extratores estudados foram submetidos à correlação linear. As análises estatísticas foram realizadas com o Sistema de Análise Estatística para Microcomputadores - SANEST (Zonta et al., 1984).

\section{RESULTADOS E DISCUSSÃO}

Observa-se que, independentemente do material de origem e dos atributos dos solos (Quadro 1), a distribuição do $\mathrm{P}$ nas diferentes frações (Figuras $1 \mathrm{e}$ 2), em presença ou ausência do SFT, foi muito similar.

$\mathrm{O} \mathrm{P}$ adicionado ao solo aumentou, significativamente, nos quatro tipos de solos a fração $P$ inorgânico (Pi NaOH 0,1 $\mathrm{mol} \mathrm{L}^{-1}$ ), que é a fração moderadamente lábil, quimiossorvida aos óxidos e à caulinita (Hedley et al., 1982). Aumentaram significativamente, mas em menor grandeza, as frações extraídas pelo bicarbonato $(\mathrm{Pi} \mathrm{NaHCO}$ ) e pela resina trocadora de ânions (Pi RTA), as quais compõem o $\mathrm{P}$ lábil do solo. A labilidade da fração $\mathrm{Pi} \mathrm{NaHCO}_{3}$ é relatada por muitos autores e indica que os teores extraídos pela RTA não representam todo o $\mathrm{P}$ disponível do solo (Tiessen et al., 1994; Linquist et al., 1997; Guo \& Yost, 1998). Devido à grande importância da disponibilidade para as plantas das frações inorgânicas e orgânicas extraídas por $\mathrm{NaHCO}_{3}$, vários pesquisadores sugeriram que essas formas fossem incluídas como índices de disponibilidade de $\mathrm{P}$ em sistemas com baixa disponibilidade e baixa adição de fertilizantes (Adepetu \& Corey, 1976; Sharpley et al., 1987; Tiessen et al., 1994;

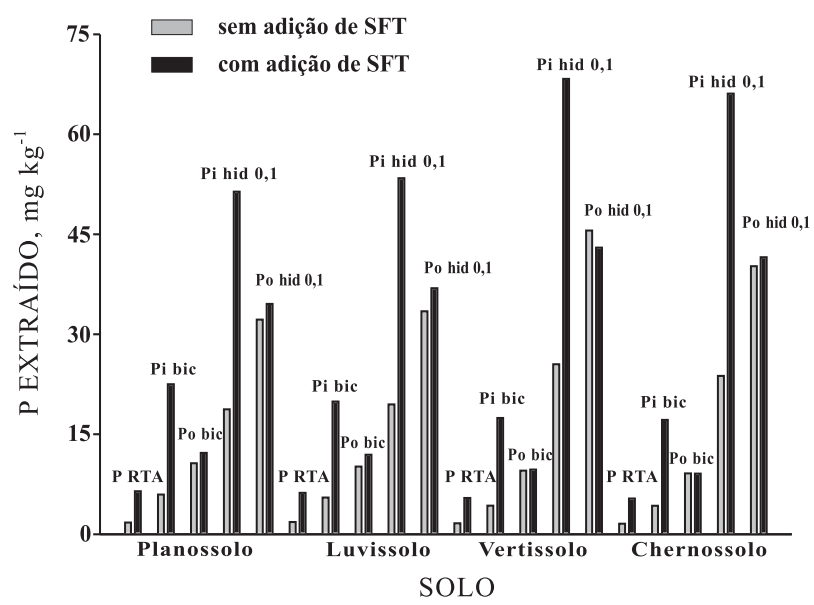

Figura 1. Teores de fósforo nas frações de amostras do Planossolo, Luvissolo, Vertissolo e Chernossolo sem e com a adição de fósforo (SFT). P-RTA: P-Resina; Pi-Bic: Pi-Bicarbonato; Po-bic: Po-Bicarbonato; Pi-hid 0,1: Pi-hidróxido 0,1 $\mathrm{mol} \mathrm{L}^{-1}$; Po-hid 0,1: Po-hidróxido 0,1 $\mathrm{mol} \mathrm{L}^{-1}$.

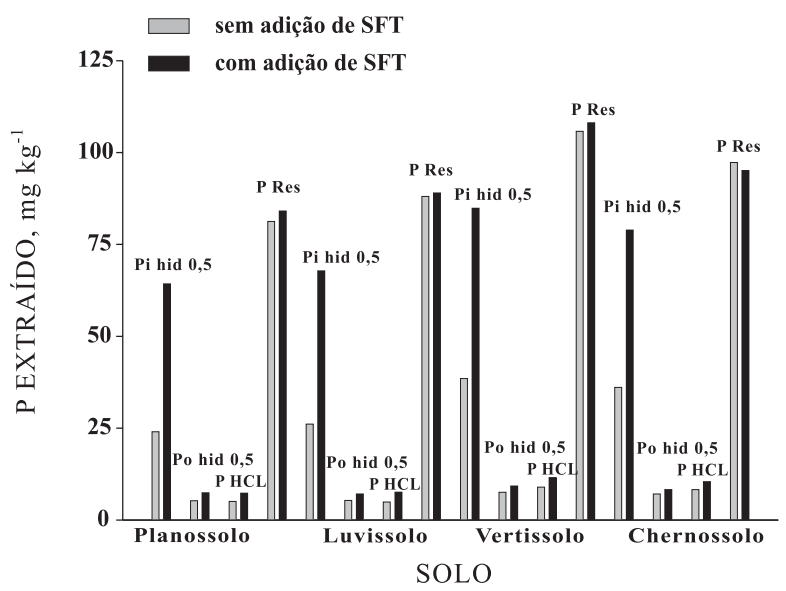

Figura 2. Teores de fósforo nas frações de amostras do Planossolo, Luvissolo, Vertissolo e Chernossolo sem e com a adição de fósforo (SFT). Pi-hid 0,5: Pi-hidróxido 0,5 mol L ${ }^{-1}$; Po-hid 0,5: Po-hidróxido $0,5 \mathrm{~mol} \mathrm{~L}^{-1}$; P-HCl; P-Res: $\mathrm{P}$ residual.

Maroko et al., 1999). Nos quatro solos, verificou-se também incremento das frações extraídas com $\mathrm{NaOH}$ $0,5 \mathrm{~mol} \mathrm{~L}^{-1}$, que são frações orgânicas e inorgânicas de P fisicamente protegidas no interior de microagregados (Cross \& Schlesinger, 1995) e não disponíveis às plantas.

Em todos os solos, independentemente da adição do SFT, observou-se que a maior parte do P encontrase na fração residual (Figura 2). Essa fração, considerada não-lábil, é representada por formas inorgânicas e orgânicas de alta recalcitrância, que podem ser lentamente disponibilizadas às plantas (Gatiboni et al., 2005). A análise desses resultados revela que $\mathrm{o} P$ recentemente adicionado às amostras dos solos incrementou, principalmente, a fração moderadamente lábil (Pi NaOH 0,1 mol L ${ }^{-1}$ ) e, em menor quantidade, as frações que compõem o P lábil do solo (Pi NaHCO 3 e Pi RTA).

$\mathrm{O} P$ acumulado pelas plantas de arroz relacionouse significativamente com as frações de $\mathrm{P}$ extraídas pela RTA (Quadro 2) e com a fração extraída com o $\mathrm{NaHCO}_{3}$ (Quadro 2), indicando que essas duas frações são lábeis e disponíveis para as plantas. Esses resultados confirmam observações de Saleque et al. (2004), que constataram que o $\mathrm{P}$ acumulado por plantas de arroz em solos alagados foi associado ao $\mathrm{P}$ lábil e à fração moderadamente lábil ( $\mathrm{Pi} \mathrm{NaOH} 0,1)$. Por outro lado, Pheav et al. (2005) observaram que a sucessão de cultivos de arroz provocou a diminuição das frações consideradas lábil, moderadamente lábil e não-lábil, sugerindo que, sob condições de alagamento, todas as frações de $\mathrm{P}$ fornecem o nutriente indireta ou diretamente às plantas. Neste trabalho, constatou-se que também as frações orgânicas (Po $\mathrm{NaHCO}_{3}$ ) e inorgânicas (Pi NaOH $0,1 \mathrm{~mol} \mathrm{~L}^{-1}$ ) apresentaram relação com o $\mathrm{P}$ acumulado pelas plantas de arroz (Quadro 2). 
A resina trocadora de íons e o extrator Mehlich-1 são utilizados pelos laboratórios de análises de solos para avaliar o $\mathrm{P}$ disponível para a cultura do arroz irrigado, por alagamento. Os teores de $\mathrm{P}$ extraídos pela resina e pelo extrator Mehlich-1 relacionaram-se significativamente com o teor de $\mathrm{P}$ nas frações lábeis e moderadamente lábeis dos solos (Quadro 3). Logo, os resultados obtidos indicam que essas frações de $\mathrm{P}$ extraídas pelos métodos da resina e Mehlich-1 relacionam-se com as absorvidas pelas plantas. Isso talvez explique o mesmo coeficiente de correlação com o P acumulado pelas plantas de arroz (Quadro 2).

Quadro 2. Coeficientes de correlação linear simples (r) entre os teores de $P$ nas frações e o $P$ acumulado pelas plantas de arroz

\begin{tabular}{lc}
\hline \multicolumn{1}{c}{ Fração } & P acumulado \\
\hline $\mathrm{RTA}$ & $0,94^{* *}$ \\
$\mathrm{Pi} \mathrm{NaHCO}$ & $0,96^{* *}$ \\
$\mathrm{Po} \mathrm{NaHCO}_{3}$ & $0,73^{*}$ \\
$\mathrm{Pi} \mathrm{NaOH} \mathrm{0,1} \mathrm{mol} \mathrm{L}$ & $0,73^{*}$ \\
Mehlich-1 & $0,94^{* *}$ \\
\hline
\end{tabular}

$* \mathrm{e}^{* *}$ : significativo a 5 e $1 \%$, respectivamente.

Quadro 3. Coeficientes de correlação linear simples (r) entre os teores de $P$ nas frações e o $P$ extraído pela Resina e pelo extrator de Mehlich-1

\begin{tabular}{|c|c|c|}
\hline \multirow{2}{*}{ Fração } & \multicolumn{2}{|c|}{ Extrator } \\
\hline & Resina (RTA) & Mehlich-1 \\
\hline $\mathrm{Pi} \mathrm{NaHCO}_{3}$ & $0,99^{* *}$ & $0,82^{*}$ \\
\hline $\mathrm{Po} \mathrm{NaHCO}_{3}$ & $0,80^{*}$ & $0,71^{*}$ \\
\hline $\mathrm{Pi} \mathrm{NaOH} 0,1 \mathrm{~mol}^{-1}$ & $0,90 * *$ & $0,90 * *$ \\
\hline Po $\mathrm{NaOH} 0,1 \mathrm{~mol} \mathrm{~L}^{-1}$ & 0,14 & 0,03 \\
\hline $\mathrm{Pi} \mathrm{NaOH} 0,5 \mathrm{~mol}^{-1}$ & $0,89^{* *}$ & $0,83^{*}$ \\
\hline Po $\mathrm{NaOH} 0,5 \mathrm{~mol} \mathrm{~L}^{-1}$ & $0,58^{*}$ & $0,53^{*}$ \\
\hline $\mathrm{HCl}$ & 0,44 & 0,36 \\
\hline
\end{tabular}

${ }^{*} \mathrm{e}^{* *}$ : significativo a 5 e $1 \%$, respectivamente.

\section{CONCLUSÕES}

1. O P absorvido pelas plantas de arroz correlacionou-se signicativamente com os teores das frações de $\mathrm{P}$ correspondentes ao $\mathrm{P}$ lábil (Pi RTA e Pi $\mathrm{NaHCO}_{3}$ ) e também com os teores nas frações do $\mathrm{P}$ moderadamente lábil ou do não-lábil $\left(\mathrm{NaOH} 0,1 \mathrm{~mol} \mathrm{~L}^{-1}\right.$ e $\mathrm{NaOH} 0,5 \mathrm{~mol} \mathrm{~L}^{-1}$ ).

2. Os teores de P extraídos das amostras dos solos pela resina e por Mehlich-1 correlacionaram-se com os teores de $\mathrm{P}$ extraídos nas frações lábil e moderadamente lábil, sendo o grau de associação com essas frações maior para a resina (Pi RTA).

3. $\mathrm{O} P$ recentemente adicionado às amostras dos solos na forma de SFT incrementou, principalmente, a fração moderadamente lábil ( $\mathrm{Pi} \mathrm{NaOH} 0,1 \mathrm{~mol} \mathrm{~L}^{-1}$ ) e, em menor proporção, as frações que compõem o $\mathrm{P}$ lábil do solo (Pi $\mathrm{NaHCO}_{3}$ e Pi RTA).

\section{LITERATURA CITADA}

ADEPETU, J.A. \& COREY, R.B. Organic phosphorus as a predictor of plant-available phosphorus in soils of Southern Nigeria. Soil Sci., 122:159-164, 1976.

BROOKES, P.C. \& POWLSON, D.S. Preventing phosphorus losses during perchloric acid digestion of sodium bicarbonate soil extracts. J. Sci. Food Agric., 32:671-674, 1981.

CHANG, S.C. \& JACKSON, M.L. Fractionation of soil phosphorus. Soil Sci., 84:133-144, 1957.

CONDRON, L.M.; GOH, K.M. \& NEWMAN, R.H. Nature and distribution of soil phosphorus as revealed by a sequential extraction method followed by $\mathrm{P}^{31}$ nuclear magnetic resonance analysis. J. Soil Sci., 36:199-207, 1985.

COUNCE, P.A.; KEISLING, T.C. \& MITCHELL, A.J. A uniform, objective, and adptative system for expressing rice development. Crop Sci., 40:436-443, 2000.

CROSS, A.F. \& SCHLENSIGER, H.A. A literature review and evaluation of the Hedley fractionation: Applications to the biochemical cycle of soil phosphorus in natural ecosystems. Geoderma, 64:197-214, 1995.

DICK, W.A. \& TABATABAI, M.A. Determination of orthophosphate in aqueous solutions containing labile organic and inorganic phosphorus compounds. J. Environ. Qual., 6:82-85, 1977.

EMPRESA BRASILEIRA DE PESQUISA AGROPECUÁRIA EMBRAPA. Centro Nacional de Pesquisa de Solos. Sistema brasileiro de classificação de solos. 2.ed. Rio de Janeiro, 2006. 306p.

GATIBONI, L.C.; SANTOS, D.R.; ANGHINONI, I. \& KAMINSKI, J. Phosphorus forms and availability assessed by 31P-NMR in successive cropped soil. Comm. Soil Sci. Plant Anal., 36:2625-2640, 2005.

GRANDE, M.A.; CURI, N. \& QUAGGIO, J.A. Disponibilidade de fósforo pelos extratores de Mehlich e Resina, em solos cultivados com arroz irrigado. R. Bras. Ci. Solo, 10:45-50, 1986.

GUO, F. \& YOST, R.S. Partitioning soil phosphorus into three discrete pools of differing availability. Soil Sci., 163:822833, 1998. 
HEDLEY, M.J.; STEWART, J.W.B. \& CHAUHAN, B.S Changes in inorganic and organic soil phosphorus fractions induced by cultivation practices and by laboratory incubations. Soil Sci. Soc. Am. J., 46:970-976, 1982.

LINQUIST, B.A.; SINGLETON, P.W. \& CASSMAN, K.G. Inorganic and organic phosphorus dynamics during a buildup decline of avaliable phosphorus in a Ultisol. Soil Sci., 162:254-264, 1997.

MAROKO, J.B.; BURESH, R.J. \& SMITHSON, P.C. Soil phosphorus fractions in unfertilized fallow-maize systems on two tropical soils. Soil Sci. Soc. Am. J., 63:320-326, 1999.

MEHRA, O.P. \& JACKSON, M.L. Iron oxide removal from soils and clay by a dithionite-citrate system buffered with sodium bicarbonate. Clays Clay Miner., 7:317-327, 1960

MURPHY, J. \& RILLEY, J.P. A modified single solution method for the determination of phosphate in natural waters. Anal. Chim. Acta, 27:31-36, 1962.

PHEAV, S.; BELL, R.W.; KIRK, G.J.D. \& WHITE, P.F. Phosphorus cycling in rainfed lowland rice ecosystems on sandy soils. Plant Soil, 269:89-98, 2005.

SCHWERTMANN, U. Differenzierung der eisenoxide des bodens durch extraction mit ammoniumoxalat-losung. Z. Pflanzenernähr Bodenk, 105:194-202, 1964.
SALEQUE, M.A.; NAHER, U.A.; ISLAM, A.; PATHAN, A.B.M.B.U.; HOSSAIN, A.T.M.S. \& MEISNER, C.A. Inorganic and organic phosphorus fertilizer effects on the phosphorus fractionation in wetland rice soils. Soil Sci. Soc. Am. J., 68:1635-1644, 2004.

SILVA, R.J. Metodologia de avaliação da disponibilidade de fósforo em solos alagados. Pelotas, Universidade Federal de Pelotas, 1996. 65p. (Tese de Mestrado)

SHARPLEY, A.N.; TIESSEN, H. \& COLE, C.V. Soil phosphorus forms extracted by soil tests as a function of pedogenesis. Soil Sci. Soc. Am. J., 51:362-365, 1987.

TEDESCO, M.J.; GIANELLO, C.; BISSANI, C.A.; BOHNEN, H. \& VOLKWEISS, S.J. Análises de solo, plantas e outros materiais. 2.ed. Porto Alegre, Universidade Federal do Rio Grande do Sul, 1995. 174p. (Boletim Técnico de Solos, 5)

TIESSEN, H.; STEWART, W.B. \& COLE, C.V. Pathways of phosphorus transformations in soils of differing pedogenesis. Soil Sci. Soc. Am. J., 48:853-858, 1994.

UNITED STATES ENVIRONMENTAL PROTECTION AGENCY - USEPA. Methods of chemical analysis for water and wastes. Cincinnati, 1971.

ZONTA, E.P.; MACHADO, A.A. \& SILVEIRA, J.P. Sistema de análise estatística para microcomputadores (SANEST). Pelotas, Universidade Federal de Pelotas, 1984. 151p. 